\title{
Enhancement of the magnetic modulation of surface plasmon polaritons in $\mathrm{Au} / \mathrm{Co} / \mathrm{Au}$ films
}

\author{
Diana Martín-Becerra, ${ }^{1, a)}$ Juan B. González-Díaz, ${ }^{1}$ Vasily V. Temnov, ${ }^{2}$ Alfonso Cebollada, ${ }^{1}$ \\ Gaspar Armelles, ${ }^{1}$ Tim Thomay, ${ }^{3}$ Alfred Leitenstorfer, ${ }^{3}$ Rudolf Bratschitsch, ${ }^{3}$ \\ Antonio García-Martín, ${ }^{1}$ and María Ujué González ${ }^{1}$ \\ ${ }^{1}$ Instituto de Microelectrónica de Madrid (IMM), CNM-CSIC, Isaac Newton 8, PTM, E-28760 Tres Cantos, \\ Madrid, Spain \\ ${ }^{2}$ Department of Chemistry, Massachusetts Institute of Technology, Cambridge, Massachusetts 02139, USA \\ ${ }^{3}$ Department of Physics and Center for Applied Photonics, University of Konstanz, D-78457 \\ Konstanz, Germany
}

(Received 30 August 2010; accepted 15 October 2010; published online 5 November 2010)

\begin{abstract}
The deposition of a dielectric overlayer on top of $\mathrm{Au} / \mathrm{Co} / \mathrm{Au}$ multilayer films can significantly enhance the magnetic field induced modulation of the surface plasmon polariton (SPP) wave vector. This enhancement is analyzed as a function of the thickness of the dielectric overlayer and the associated SPP electromagnetic field confinement and redistribution. The decrease in SPP propagation distance is taken into account by analyzing an adequate figure of merit. (C) 2010 American Institute of Physics. [doi:10.1063/1.3512874]
\end{abstract}

Surface plasmon polaritons (SPPs) are evanescent waves that propagate along a metal-dielectric interface. They can be laterally confined below the diffraction limit using subwavelength metal structures, rendering them attractive for the development of miniaturized optical devices. ${ }^{1}$ Passive plasmonic circuits have been extensively proposed and analyzed in the last decade ${ }^{2,3}$ but active plasmonic configurations are needed to achieve nanophotonic devices with advanced functionalities. This requires systems where the plasmon properties can be rapidly modulated by an external parameter, such as temperature, ${ }^{4,5}$ voltage, ${ }^{6,7}$ or electromagnetic radiation. ${ }^{8-10}$

The magnetic field is another interesting candidate to control SPPs, since it is able to modify their dispersion relation. ${ }^{11-14}$ For a magnetic field $B$ applied along the interface and perpendicular to the SPP propagation direction, the SPP wave vector $k_{s p}$ can be described as

$$
k_{s p}(B)=k_{s p}^{0}+\Delta k_{s p}(B),
$$

where $k_{s p}^{0}$ is the SPP wave vector without applied magnetic field. The modulation term, $\Delta k_{s p}(B)$, is proportional to the off-diagonal elements of the dielectric tensor $\left(\varepsilon_{i j}, i \neq j\right)$, which depend on the magnetic field. ${ }^{11-13}$ In noble (diamagnetic) metals those elements are small but can be orders of magnitude larger in ferromagnetic metals, where $\varepsilon_{i j}$ depends on the sample magnetization. Since optical absorption in ferromagnetic metals heavily damps surface plasmon propagation, we have developed hybrid magnetoplasmonic multilayer structures consisting of low loss plasmonic noble metal films and magneto-optical ferromagnetic compounds. ${ }^{14-16}$ Using magnetoplasmonic microinterferometers, we have recently demonstrated the modulation of SPP wave vector in hybrid $\mathrm{Au} / \mathrm{Co} / \mathrm{Au}$ multilayers. ${ }^{16}$ However, the practical application of a magnetoplasmonic interferometer as an optical switch requires further optimization of multilayer films to achieve the maximum possible SPP wave vector modulation.

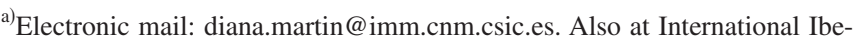
rian Nanotechnology Laboratory, 4710-229 Braga, Portugal.
}

In this paper, we show that the deposition of a dielectric layer on top of a metallic multilayer provides a sevenfold increase of $\Delta k_{s p}$. We analyze in detail the performance of the magnetoplasmonic interferometers in the presence of this overlayer.

For a very thin Co layer, an analytical expression for the modulation of $k_{s p}$ in an $\mathrm{Au} / \mathrm{Co} / \mathrm{Au}$ multilayer system (as in Fig. 1 but with an infinitely thick dielectric overlayer) can be obtained $^{16}$

$$
\Delta k_{s p}(h) \approx \frac{2 t_{\mathrm{Co}} k_{0}^{2} \varepsilon_{d}^{2}}{-\varepsilon_{\mathrm{Au}}} \frac{i \varepsilon_{y z}^{\mathrm{Co}}}{\varepsilon_{\mathrm{Co}}} e^{-2 h k_{z}^{\mathrm{Au}}},
$$

where we have considered $\left|\varepsilon_{d}\right| \ll\left|\varepsilon_{\mathrm{Au}}\right|$, being $\varepsilon_{\mathrm{Au}}$ the gold dielectric permittivity and $\varepsilon_{d}$ the dielectric constant of the dielectric overlayer; $h$ is the upper gold layer thickness and therefore stands for the cobalt layer position; $t_{\mathrm{Co}}$ is the thickness of the cobalt layer; $\varepsilon_{\mathrm{Co}}$ and $\varepsilon_{y z}^{\mathrm{Co}}$ represent the diagonal and nondiagonal elements of the complex dielectric permittivity tensor for cobalt; $k_{z}^{\mathrm{Au}}$ provides the SPP field penetration in the metal layer; ${ }^{1,2}$ and $k_{0}$ is the wave vector of light. As it can be seen, the SPP wave vector modulation is proportional to the square of the permittivity of the dielectric layer on top of the metallic multilayer. Therefore, placing dielectric layers with higher $\varepsilon_{d}$ constitutes a simple means of increasing $\Delta k_{s p}$. Covering the metallic multilayer with an infinitely thick di-

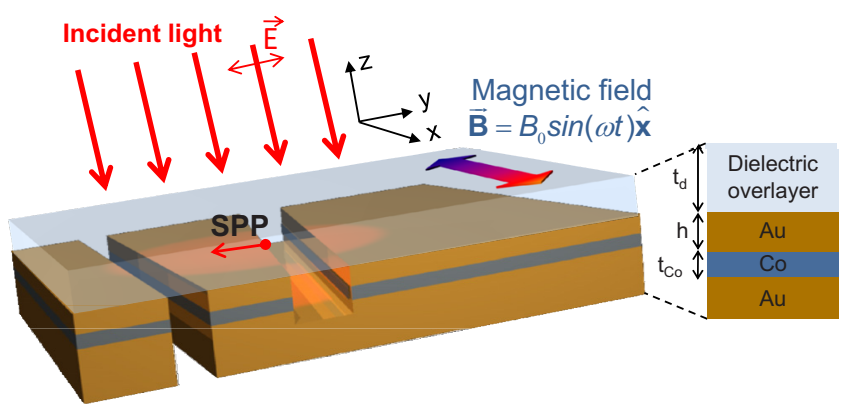

FIG. 1. (Color online) Sketch of the magnetoplasmonic interferometer consisting of a metallic $\mathrm{Au} / \mathrm{Co} / \mathrm{Au}$ trilayer covered by a thin dielectric film. 


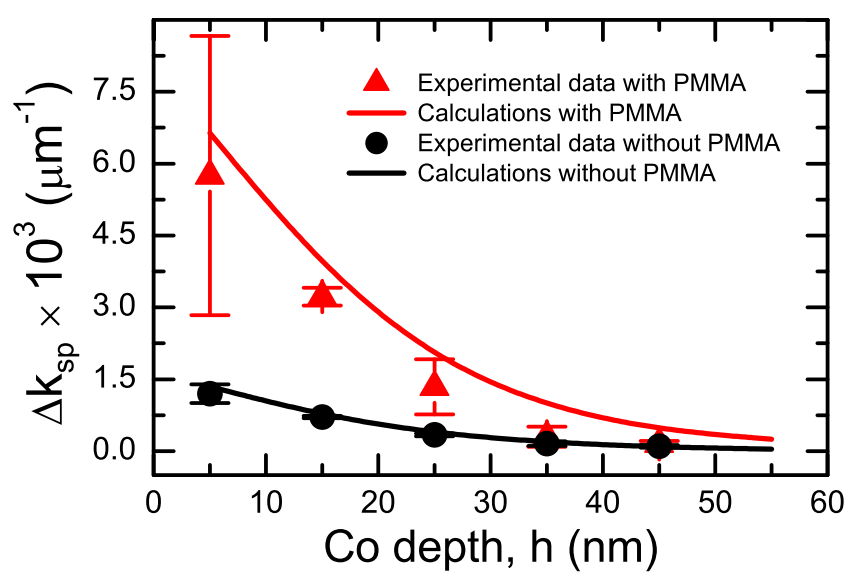

FIG. 2. (Color online) Evolution of the SPP wave vector modulation with the Co layer position for a set of metallic multilayers covered with $60 \mathrm{~nm}$ of PMMA and without coverage.

electric other than air is not experimentally feasible but we can analyze the effect of adding thin dielectric overlayers, which can be seen as adding an effective medium with intermediate dielectric constant. ${ }^{17}$

To that end, in the experiment we have employed the same configuration as in Ref. 16, i.e., plasmonic microinterferometers in $\mathrm{Au} / \mathrm{Co} / \mathrm{Au}$ multilayers, spin-coating the metallic multilayer with a $60 \mathrm{~nm}$ film of polymethyl methacrylate (PMMA) ( $\mathrm{n}=1.49)$. The Co layer thickness, $t_{\mathrm{Co}}=6 \mathrm{~nm}$ is equal for all samples, as well as the total metal thickness, $200 \mathrm{~nm}$; the Co layer position, $h$, is varied from 5 to $45 \mathrm{~nm}$. Figure 1 shows a sketch of the system geometry. The plasmonic microinterferometer consists of a tilted slit-groove pair. ${ }^{18}$ When illuminating the interferometer with a $p$-polarized laser $\left(\lambda_{0}=633 \mathrm{~nm}\right)$, light directly transmitted through the slit, $I_{r}$, interferes with SPPs excited in the groove and converted into free space radiation in the slit, $I_{s p}$. Due to the tilted slit-groove arrangement the light collected at the back side of the slit exhibits a pronounced interference pattern along the slit axes. The interference term is given by $2 \sqrt{I_{s p}} \sqrt{I_{r}} \cos \left[k_{s p} d(x)+\phi_{0}\right]$, where $d(x)$ stands for groove-slit distance. An external periodic $(1.4 \mathrm{kHz})$ magnetic field high enough to saturate the sample $(B \sim 20 \mathrm{mT})$ changes the magnitude of $k_{s p}$ and thus shifts the plasmonic interference pattern. This leads to a variation in the intensity synchronous with the applied magnetic field at each point of the slit. Assuming $\operatorname{Im}\left(\Delta k_{s p}\right) \approx 0$, this intensity variation can be expressed as $\Delta I=-4 \sqrt{I_{s p}} \sqrt{I_{r}} \Delta k_{s p} d \sin \left[k_{s p}^{0} d(x)+\phi_{0}\right]$. Dividing the contrast of $\Delta I$ by the contrast of the optical interference term $\left(4 \sqrt{I_{s p}} \sqrt{I_{r}}\right)$ gives us $\Delta k_{s p} d$ from where, $d$ being known, we can extract $\Delta k_{s p}$.

Figure 2 compares $\Delta k_{s p}$ for $\mathrm{Au} / \mathrm{Co} / \mathrm{Au}$ trilayers with different $h$ coated by a $60 \mathrm{~nm}$ layer of PMMA (triangles) with data without coating (dots). The SPP wave vector modulation decays exponentially with $h$, as expected from Eq. (2). ${ }^{16}$ More interestingly, for every Co layer position, $\Delta k_{s p}$ is higher for the trilayers covered by PMMA, corroborating the theoretical prediction about the SPP modulation enhancement caused by the dielectric layer.

We have compared experimental data with theoretical modeling based on a transfer matrix formalism that incorporates magneto-optical effects. ${ }^{14,15}$ In the calculations we used values for $\varepsilon_{\mathrm{Au}}, \varepsilon_{\mathrm{Co}}$, and $\varepsilon_{y z}^{\mathrm{Co}}$ experimentally determined for
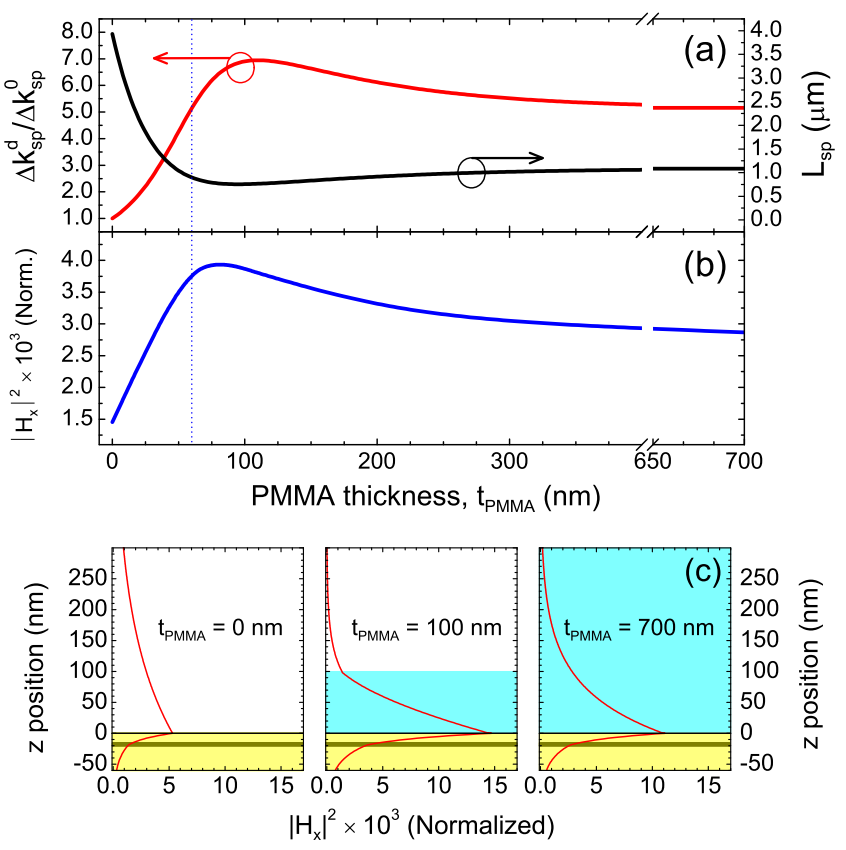

FIG. 3. (Color online) (a) Calculated modulation enhancement (left axis) and SPP propagation distance (right axis) as a function of the thickness of PMMA coverage for an $\mathrm{Au} / \mathrm{Co} / \mathrm{Au}$ trilayer with $h=15 \mathrm{~nm}$. (b) Normalized magnetic field intensity calculated at the center of the Co layer. The dashed line marks the thickness value used experimentally, $60 \mathrm{~nm}$. (c) Normalized magnetic field intensity distribution along the vertical direction for three PMMA thicknesses: 0, 100, and $700 \mathrm{~nm}$. The different background colors account for the different materials in the structure.

our films by ellipsometry and polar Kerr spectroscopy. ${ }^{15}$ The obtained results for the metallic layer covered by $60 \mathrm{~nm}$ of PMMA (red line) and without coverage (black line) are also plotted in Fig. 2, and show an excellent agreement with the experimental values.

The increase in the magnetoplasmonic SPP modulation due to the addition of a dielectric overlayer can be quantified in terms of the enhancement factor $\Delta k_{s p}^{d} / \Delta k_{s p}^{0}$, with $\Delta k_{s p}^{d}$ the SPP wave vector modulation for the system covered with a dielectric film and $\Delta k_{s p}^{0}$ the modulation for the uncovered system. From the data shown in Fig. 2, we infer an enhancement factor of 4.5 for a $60 \mathrm{~nm}$ overlayer thickness when $h=5-15 \mathrm{~nm}$.

It is important to choose the optimum thickness of the dielectric overlayer, which delivers the maximum possible enhancement factor. Figure 3(a) shows the calculated evolution of the modulation enhancement factor as a function of the PMMA thickness for a trilayer with $h=15 \mathrm{~nm}$. Instead of a monotonous increase with the PMMA thickness $\Delta k_{s p}^{d}$ goes through a maximum at around $110 \mathrm{~nm}$, where the enhancement reaches a value of 7 , and then decreases until reaching a saturation value of 5 around $650 \mathrm{~nm}$. This behavior is related to the SPP electromagnetic field redistribution caused by the presence of a dielectric overlayer [see the three panels in Fig. 3(c)]: ${ }^{17}$ a thin dielectric layer causes a waveguiding effect providing the strongest confinement of SPP electromagnetic field for $t_{\mathrm{PMMA}} \sim 100 \mathrm{~nm}$. As a consequence, the normalized SPP magnetic field intensity at the position of the cobalt layer [Fig. 3(b)] exhibits a nonmonotonous behavior similar to that of the enhancement factor, supporting our explanation. These two curves are not exactly equivalent because $\Delta k_{s p}$ also depends on $\varepsilon_{d}$ [Eq. (2)], which effectively increases as the thickness of the overlayer grows. 


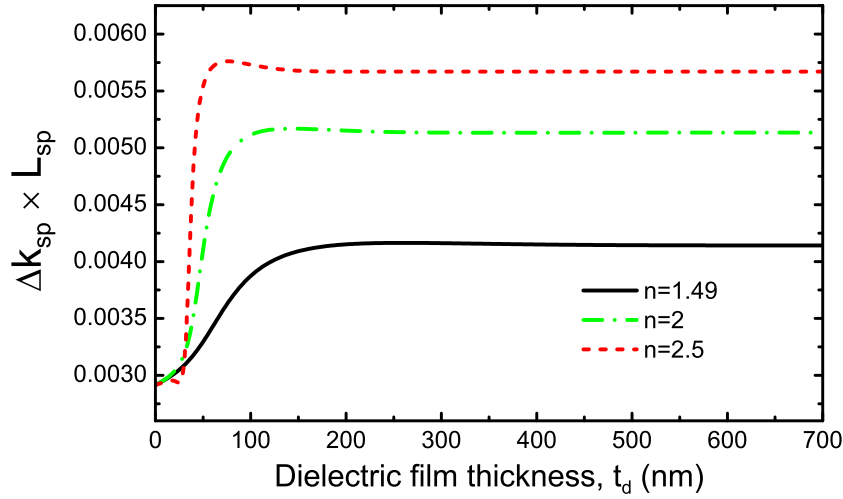

FIG. 4. (Color online) Figure of merit $\Delta k_{s p} \times L_{s p}$ for an $\mathrm{Au} / \mathrm{Co} / \mathrm{Au}$ trilayer with $h=15 \mathrm{~nm}$ and three different dielectrics.

It is well known that the addition of a dielectric overlayer decreases the propagation distance in SPPs, $L_{s p}{ }^{17}$ Figure 3(b) shows this effect in our system, where we observe that the reduction on $L_{s p}$ is indeed quite strong. This could preclude the application of these dielectric covered Au/ $\mathrm{Co} / \mathrm{Au}$ multilayers in actual devices, so a compromise between the modulation enhancement and the propagation distance of the SPP has to be achieved. In fact, for magnetoplasmonic interferometers the full intensity modulation depth is proportional to the product $\Delta k_{s p} \times d$. Therefore, the product $\Delta k_{s p} \times L_{s p}$ represents an appropriate figure of merit to analyze the performance of this system. ${ }^{16}$ In Fig. 4 we plot this product as a function of the dielectric film thickness for metallic trilayers covered by dielectric layers of different refractive indexes. The decrease in $L_{s p}$ is compensated by a much stronger rise in $\Delta k_{s p}$ resulting into the overall increase for the figure of merit as a function of dielectric overlayer thickness $t_{d}$. Moreover, this increase is higher for materials with a higher refractive index.

To summarize, we have demonstrated that the deposition of a dielectric overlayer on top of noble/ferromagnetic metal multilayers leads to a significant enhancement of the magnetic field induced modulation of the SPP wave vector. The analysis of the figure of merit shows that the modulation depth of a magnetoplasmonic switch can be increased despite of the strong reduction in SPP propagation length, which allows to reduce the size of the device. Therefore, this finding represents an essential step toward miniaturization of active magnetoplasmonic devices.

We acknowledge funding from the EU (Grant No. NMP3-SL-2008-214107 Nanomagma), the Spanish MICINN ("MAGPLAS" Grant No. MAT2008-06765-C02-01/NAN, "FUNCOAT" CONSOLIDER INGENIO 2010 Grant Nos. CSD2008-00023, and MAT2005-05524-C02-01), the Comunidad de Madrid ("NANOBIOMAGNET" Grant No. S2009/ MAT-1726 and "MICROSERES-CM" Grant No. S2009/ TIC-1476), CSIC (Grant No. 200850I187), and Deutsche Forschungsgemeinschaft (Grant No. TE770/1).

${ }^{1}$ W. L. Barnes, A. Dereux, and T. W. Ebbesen, Nature (London) 424, 824 (2003).

${ }^{2}$ T. W. Ebbesen, C. Genet, and S. I. Bozhevolnyi, Phys. Today 61(5), 44 (2008).

${ }^{3}$ D. K. Gramotnev and S. I. Bozhevolnyi, Nat. Photonics 4, 83 (2010).

${ }^{4}$ A. V. Krasavin and N. I. Zheludev, Appl. Phys. Lett. 84, 1416 (2004).

${ }^{5}$ J. Gosciniak, S. I. Bozhevolnyi, T. B. Andersen, V. S. Volkov, J. KjelstrupHansen, L. Markey, and A. Dereux, Opt. Express 18, 1207 (2010).

${ }^{6}$ J. A. Dionne, K. Diest, L. A. Sweatlock, and H. A. Atwater, Nano Lett. 9, 897 (2009).

${ }^{7}$ M. J. Dicken, L. A. Sweatlock, D. Pacifici, H. J. Lezec, K. Bhattacharya, and H. A. Atwater, Nano Lett. 8, 4048 (2008).

${ }^{8}$ D. Pacifici, H. J. Lezec, and H. A. Atwater, Nat. Photonics 1, 402 (2007).

${ }^{9}$ R. A. Pala, K. T. Shimizu, N. A. Melosh, and M. L. Brongersma, Nano Lett. 8, 1506 (2008).

${ }^{10}$ K. F. MacDonald, Z. L. Sámson, M. I. Stockman, and N. I. Zheludev, Nat. Photonics 3, 55 (2009).

${ }^{11}$ R. F. Wallis, J. J. Brion, E. Burstein, and A. Hartstein, Phys. Rev. B 9, 3424 (1974).

${ }^{12}$ Z. Yu, G. Veronis, Z. Wang, and S. Fan, Phys. Rev. Lett. 100, 023902 (2008).

${ }^{13}$ V. I. Belotelov, D. A. Bykov, L. L. Doskolovich, A. N. Kalish, and A. K. Zvezdin, J. Opt. Soc. Am. B 26, 1594 (2009).

${ }^{14}$ J. B. González-Díaz, A. García-Martín, G. Armelles, J. M. García-Martín, C. Clavero, A. Cebollada, R. A. Lukaszew, J. R. Skuza, D. P. Kumah, and R. Clarke, Phys. Rev. B 76, 153402 (2007).

${ }^{15}$ E. Ferreiro-Vila, J. B. González-Díaz, R. Fermento, M. U. González, A. García-Martín, J. M. García-Martín, A. Cebollada, G. Armelles, D. Meneses-Rodríguez, and E. M. Sandoval, Phys. Rev. B 80, 125132 (2009).

${ }^{16}$ V. V. Temnov, G. Armelles, U. Woggon, D. Guzatov, A. Cebollada, A. García-Martín, J. M. García-Martín, T. Thomay, A. Leitenstorfer, and R. Bratschitsch, Nat. Photonics 4, 107 (2010).

${ }^{17}$ T. Holmgaard and S. I. Bozhevolnyi, Phys. Rev. B 75, 245405 (2007).

${ }^{18}$ V. V. Temnov, K. Nelson, G. Armelles, A. Cebollada, T. Thomay, A. Leitenstorfer, and R. Bratschitsch, Opt. Express 17, 8423 (2009). 